\title{
Direct activation of caspase 8 by the proapoptotic E2 protein of HPV18 independent of adaptor proteins
}

\author{
F Thierry ${ }^{1,2}$ and C Demeret ${ }^{*, 1,3}$
}

The self-activation of initiator caspases is dependent on their oligomerization driven by interaction with the death fold domains (DFD) of adaptor proteins. Here, we show that the E2 protein of human papillomavirus type 18 triggers apoptosis by assembling cytoplasmic filaments together with caspase 8 , in which its efficient self-activation occurs. The E2 protein binds directly to the death effector domains (DED) of caspase 8 through non-DFD interaction. This interaction is independent of FADD, but it can cooperate with FADD homotypic binding to caspase 8 to induce its oligomerization; hence cell death, while it is antagonized by competitive binding of MC159 FLICE inhibitory protein. The amino-terminal domain of E2 contains a 27 amino-acid $\alpha$-helix, which is necessary and sufficient to induce caspase oligomerization and cell death. Our results provide evidence for adaptorindependent oligomerization of caspase 8, mediated by non-DFD direct interactions with the HPV18 E2 protein, thus deciphering a new pathway for caspase 8 activation.

Cell Death and Differentiation (2008) 15, 1356-1363; do:10.1038/cdd.2008.53; published online 18 April 2008

\section{Introduction}

Viruses have evolved complex strategies to interact with the cellular apoptotic machinery, and exhibit both pro- and antiapoptotic functions. Oncogenic, or 'high-risk,' genital human papillomaviruses (HR-HPV) display well-characterized antiapoptotic activities notably mediated by the E6 oncogene, which interferes with both p53- and Fas-mediated apoptosis by targeting p53 and FADD for proteasomal degradation. ${ }^{1,2} \mathrm{HPV}$ proapoptotic functions are provided by the E7 oncogene, ${ }^{3}$ as well as by the E2 transcription factor. ${ }^{4-6}$ However, the proapoptotic function of E2 is only associated with the oncogenic HR-HPV types, and not with the low-risk types LR-HPV, which are only associated with benign lesions. ${ }^{7}$ Our previous study has shown that the E2 apoptotic activity is not cell-type specific, and is mediated by the aminoterminal domain independently of its associated transcriptional functions. ${ }^{5}$ Induction of apoptosis by HR-HPV E2 proteins is linked to their cytoplasmic localization and involves activation of the apical caspase $8^{5,7}$

Caspase-mediated apoptosis depends on the activation of apical caspases that occurs by oligomerization following their recruitment by adaptor proteins to death-signaling complexes. The coordinated assembly of death complexes depends on a network of specific homotypic interactions between domains of the death fold domain family (DFD), including caspase recruitment domains (CARD), death domains (DD) and death effector domains (DED), which all consist of six $\alpha$-helical bundle structures. ${ }^{8}$ The assembly of death complexes is a tightly regulated process that provides initiator caspases with an adequate degree of oligomerization to allow their efficient self-activation. ${ }^{9-12}$

Initiator caspases are stored as latent precursors depending on DFD adaptor proteins for oligomerization leading to catalytic activation. In contrast, adaptor proteins exhibit selfoligomerization properties uncovered upon death signaling, which drive the oligomerization of apical caspases. ${ }^{9-12}$ When ectopically expressed, the FADD adaptor has been shown to spontaneously self-oligomerize in proapoptotic filaments called 'death effector filaments,' which are able to recruit and activate caspase $8 .^{13}$ In contrast, caspase 8 strictly depends on homotypic binding to DFD adaptors such as FADD to oligomerize, and hence to self-activate.

In this study, we present evidence that the HPV18 E2 protein induces caspase 8 oligomerization within cytoplasmic filamentous structures in which efficient caspase self-activation occurs, thereby inducing apoptosis. We show that E2, despite lack of a DFD domain, directly binds to the DEDcontaining prodomain of caspase 8 , mediating the recruitment of the caspase into filaments. Caspase 8 oligomerization in the presence of E2 is independent of endogenous FADD, which accordingly was found dispensable for E2-induced apoptosis, whereas siRNA-mediated silencing of the endogenous caspase 8 severely affected E2-mediated cell death. The viral MC159 vFLIP protein, which interferes with the binding of E2 to caspase 8, antagonizes filament assembly and apoptosis. A 27-amino-acids-long $\alpha$-helix of the E2 $\mathrm{N}$-terminal domain is sufficient to induce filament formation and cell death. We propose that HR-HPV E2 induces caspase 8 oligomerization in a new type of death complex, not involving

${ }^{1}$ Unité Expression Génétique et Maladies, CNRS FRE 2850, Institut Pasteur, 25 rue du Dr Roux, Paris cedex 15, France

*Corresponding author: C Demeret, Virology, Institut Pasteur, 25, rue du Dr Roux, Paris 75015, France. Tel: + 33145688526 ; Fax: + 3314568 89 66;

E-mail: cdemeret@pasteur.fr

${ }^{2}$ Current address: Institute of Medical Biology, Biopolis, Singapore 138673

${ }^{3}$ Current address: Unité postulante Génétique, Papillomavirus et Cancer Humain, Institut Pasteur, 25 rue du Dr Roux, 75724 Paris cedex 15, France

Keywords: adaptors; apoptosis; caspase 8; death fold domains; HPV18 E2

Abbreviations: CARD, caspase recruitment domains; CD, caspase domain; DD, death domain; DED, death effector domain; DFD, death fold domain; DISC, deathinducing signaling complex; HR, high risk; vFLIP, viral FLICE inhibitory protein

Received 15.10.07; revised 14.3.08; accepted 20.3.08; Edited by JM Hardwick; published online 18.4.08 
DFD interactions, thereby providing evidence for a novel adaptor-independent pathway for caspase 8 activation.

\section{Results}

Cell death follows E2 assembly into cytoplasmic filaments containing activated caspase 8 . We previously demonstrated that the E2 proteins of HR-HPVs induce apoptosis through their $\mathrm{N}$-terminal domain in all human cell types tested, ${ }^{5}$ and that this activity is linked to their cytoplasmic accumulation due to an active nucleocytoplasmic shuttling which is specific of the HR-HPV E2 proteins. ${ }^{7}$ The purpose of this study was to decipher the mechanism by which the cytoplasmic HR-HPV E2 proteins trigger apoptosis. We used two experimental systems to address this question: first, infection by recombinant adenoviruses to express GFP-fused HPV18-E2 in Saos cells, which induce apoptosis at about $24 \mathrm{~h}$ post-infection in our conditions; second, transient transfection of 293 cells with E2 expression vectors, inducing apoptosis later (between 40 and $48 \mathrm{~h}$ post-infection), thus allowing for biochemical and immunofluorescence analyses for the first $24 \mathrm{~h}$ after transfection.

The prominent role of caspase 8 as the initiator caspase of E2-induced apoptosis was confirmed using inhibitors specific for the apical caspase 8; that is, the MC159 vFLIP protein (FLICE inhibitory protein) from Molluscum contagiosum and the cowpox virus serpin crmA. Transient transfection of 293 cells with HPV18 E2 alone induced cell death, as measured by PI uptake (Figure 1a) and checked in phase contrast microscopy (Figure 1b), whereas coexpression of MC159 or crmA efficiently abrogated it, establishing that activation of the endogenous caspase 8 is essential to E2-induced cell death (Figure 1). In addition, direct silencing of the endogenous caspase 8 with specific siRNA also significantly reduced the level of E2-induced cell death (Figure 2). Altogether, these results establish that activation of the endogenous caspase 8 is the initiating event of apoptosis triggered by the HPV18 E2 protein.

Real-time microscopy of Saos cells infected by HPV18-E2 recombinant adenovirus indicated that, after an initial nuclear accumulation, GFP-E2 progressively redistributed to the cytoplasm where it assembled into filamentous structures, which consistently preceded induction of cell death (Figure 3a). Cells expressing GFP-E2 $\Delta$ NT did not show cell death, despite high levels of nuclear accumulation of the protein (Figures 1a and 3a). Immunofluorescence performed in E2-expressing cells showed that the endogenous caspase 8 colocalizes with GFP-E2 within filaments, whereas it is found diffuse in the cytoplasm of non-infected cells (NI) or cells expressing the non-apoptotic deleted E2 protein (GFP-E2 $\Delta N T$ ) (Figure 3b). Similar results were found in 293 cells either with GFP-tagged (not shown) or untagged HPV18E2 and E2 $\Delta$ NT (Supplementary Figure S1-A), confirming that assembly of filaments containing $\mathrm{E} 2$ and the endogenous caspase 8 is not cell-type specific, and does not depend on the GFP tag, as shown earlier for the E2-induced apoptosis. ${ }^{5,6}$

In E2-expressing dead cells, only the cleaved prodomain of endogenous caspase 8 was found in a detergent-insoluble
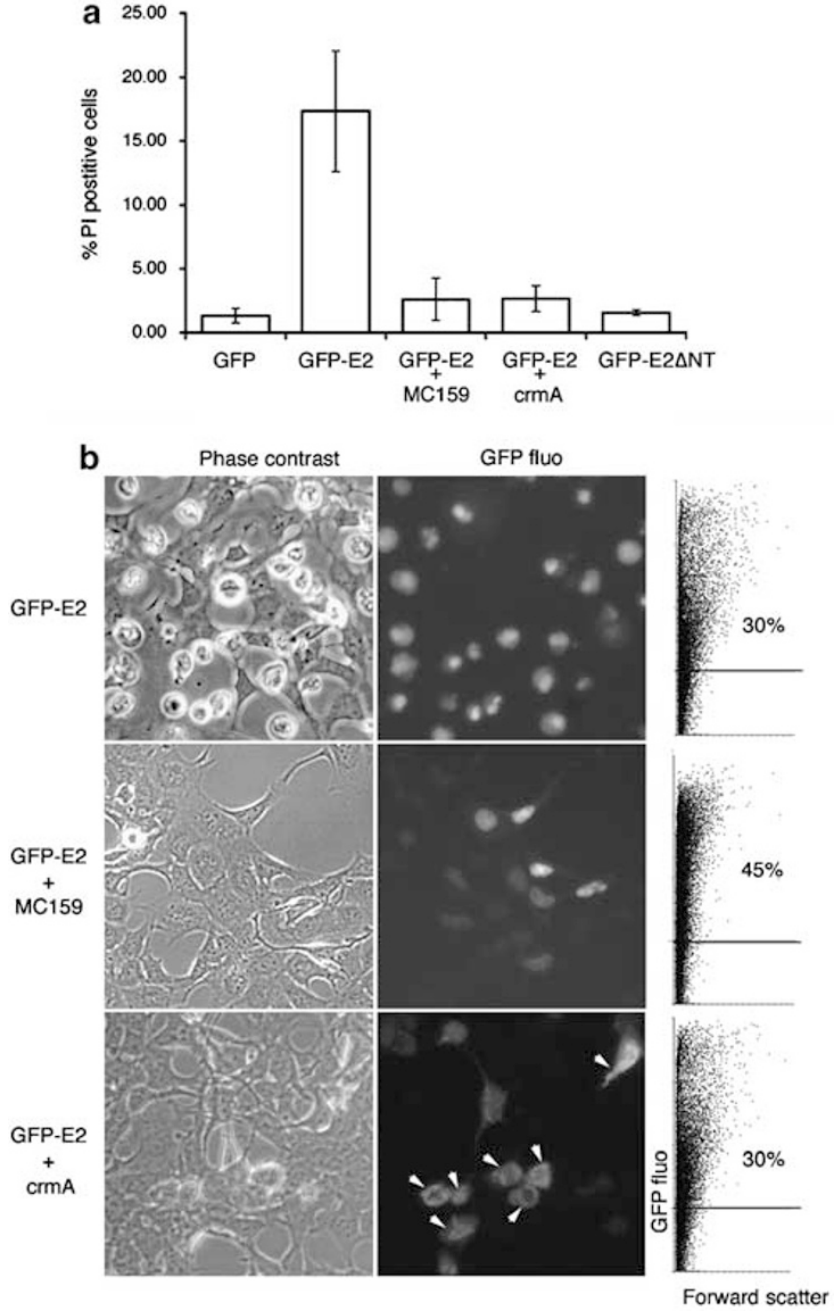

Figure 1 Caspase 8 inhibitors abrogate E2-induced apoptosis. (a) 293 cells were transfected with expression plasmids as indicated. GFP-positive dead cells were quantified by propidium iodide uptake $48 \mathrm{~h}$ after transfection. (b) Phase contrast and GFP fluorescence of 293 cells, $48 \mathrm{~h}$ after transfection with expression plasmids as indicated. Filaments containing GFP-E2 can be observed in the presence of the caspase 8 catalytic inhibitor $\mathrm{CrmA}$ (shown by arrows), but are not formed in the presence of MC159. The \% of GFP-E2-positive cells were determined by FACS and are shown for each panel

fraction of cell extracts. This coincided with the complete disappearance of the full-length caspase proform in the soluble fractions, which is the only form of endogenous caspase 8 found in living cells, either not infected or expressing E2 $\Delta \mathrm{NT}$ (Figure 3c). Self-catalytic processing of caspase 8 may therefore have occurred within filaments. Indeed, IETDase activity, which measures the enzymatic activity of caspase 8, was readily detectable in extracts from GFP-E2-expressing cells but not from NI or E2 $\Delta$ NT-expressing cells, providing a direct evidence for caspase 8 activation (Figure 3d). Filaments could be formed in the presence of the caspase inhibitor Z-VAD-fmk, indicating that filament formation is an upstream event of E2-induced apoptosis and can occur independently of caspase activity (Supplementary Figure S2). Overall, our data show that the HPV18 E2 protein triggers apoptosis through prior assembly into cytoplasmic 

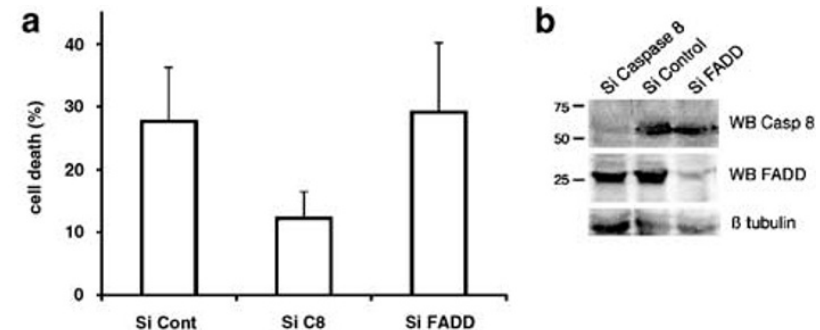

$$
\text { C }
$$

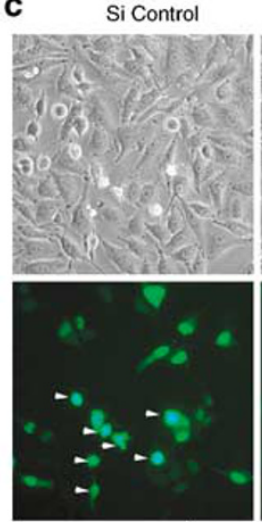

Si Caspase 8

Si FADD
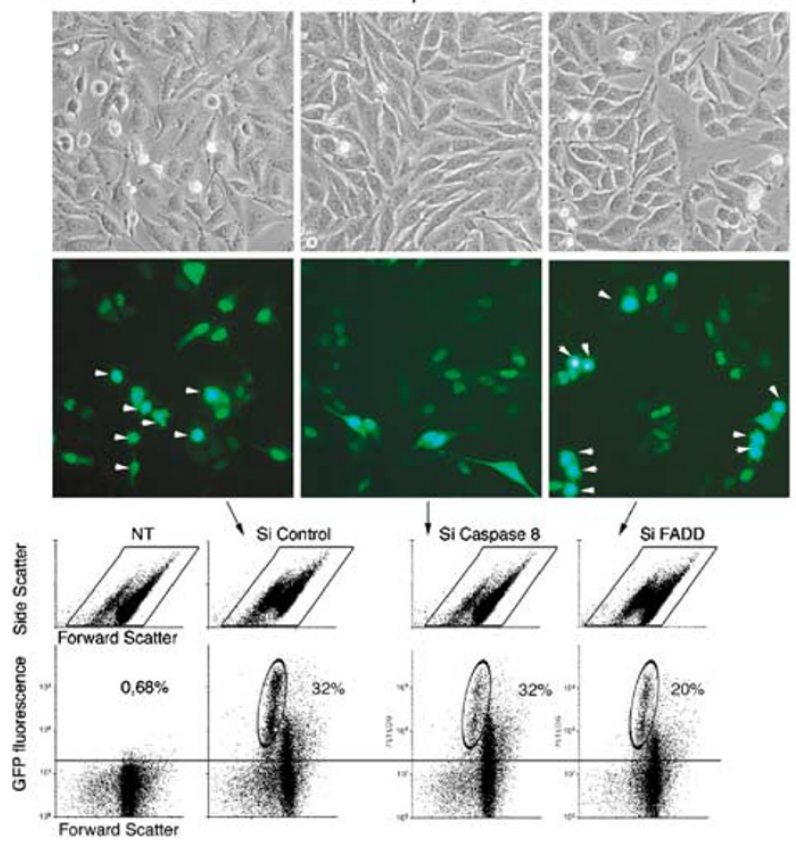

Figure 2 siRNA-mediated silencing of caspase 8 reduces E2-induced apoptosis, whereas FADD knockdown does not. (a) HeLa cells co-transfected with GFP-E2-expressing plasmid and siRNA against caspase 8 or FADD, as indicated were counted for GFP-positive dead cells $24 \mathrm{~h}$ post-transfection in three independent experiments. (b) Efficiency of siRNA-mediated caspase 8 and FADD silencing was checked by Western blots of total extracts of HeLa cells co-transfected as in A. (c) Phase contrast and GFP fluorescence of HeLa cells transfected as in A. GFP-E2-expressing dead cells are shown by white arrows. FACS analyses of the entire cell populations gated on forward and side scatters are given in the upper panels and the $\%$ of total GFP-positive cells are given in the lower panels. GFPpositive dead cells are circled and correspond to 36 and $31 \%$ of the positive cells for the control and FADD siRNAs, respectively and $13 \%$ for the C8 siRNA in this specific experiment. FACS histograms of non-transfected HeLa cells (NT) were used as reference for GFP positivity of transfected cells

filaments that efficiently recruit and activate the endogenous caspase 8.

The high-risk HPV E2-mediated cell death correlates with formation of cytoplasmic insoluble filaments. We further analyzed the relationship between E2 and caspase 8 by co-transfection in 293 cells, where ectopic expression of either E2 or wild-type caspase 8 separately did not induce cell death for at least $24 \mathrm{~h}$, during which the caspase distributed diffusely in the cytoplasm (Figure $4 \mathrm{~b}$ ) similarly to the endogenous caspase (Figure $3 \mathrm{~b}$ ). We therefore studied the relationship between ectopically coexpressed a
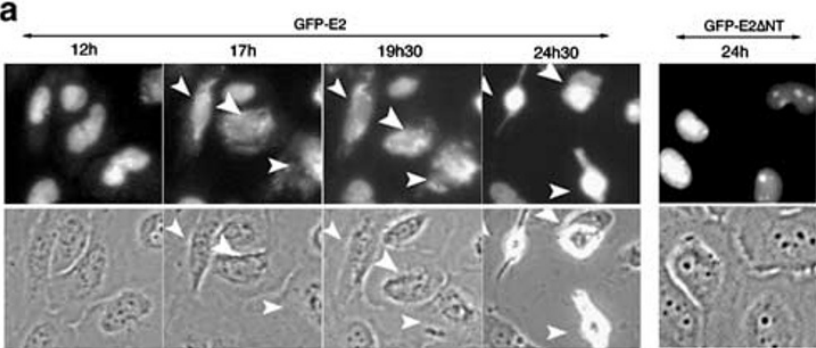

b

GFP livo

$\alpha$ C8-TR
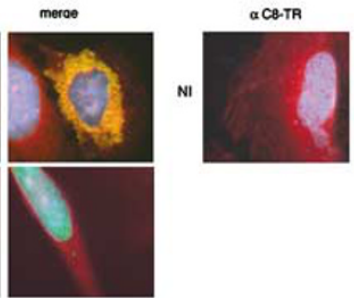

GFP-E2ANT
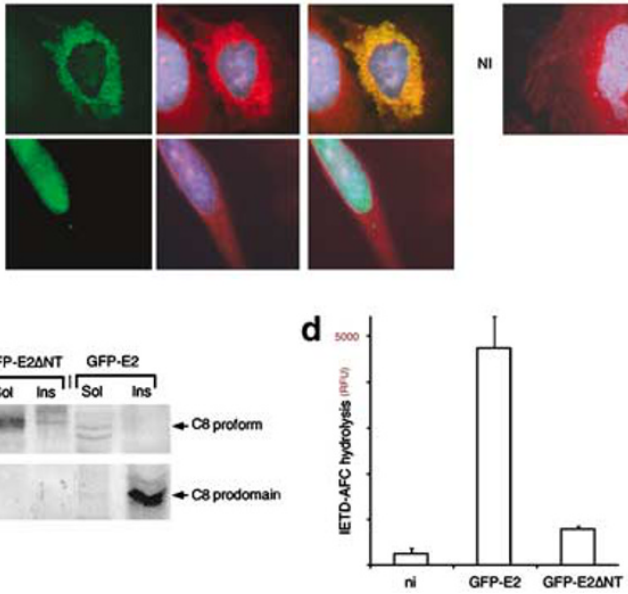

C

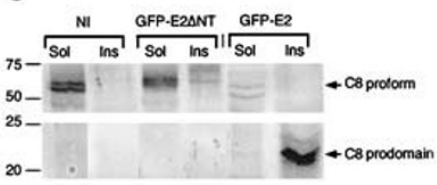

Figure 3 Formation of filaments containing E2 and caspase 8 precedes cell death. (a) Real-time microscopy was performed with Saos cells infected by recombinant adenoviruses expressing GFP-E2 or GFP-E2 $\Delta$ NT. Fluorescent and phase contrast images of the same field at different time points are shown. White arrows show dying cells. (b) Saos cells were either non-infected (NI) or infected as in A for $20 \mathrm{~h}$ and were then processed for anti-caspase 8 immunofluorescence using an antibody directed against the caspase $\mathrm{N}$-terminal prodomain, with a secondary antibody coupled to Texas-Red (TR) and DAPI staining of the nuclei. (c) Saos cells either non-infected $(\mathrm{NI})$ or infected as in (a) were extracted $30 \mathrm{~h}$ post-infection in P300 buffer containing 1\% Triton. Immunoblots were performed with the same caspase 8 antibody as in B with equivalent soluble (Sol) and insoluble (Ins) fractions. Caspase precursor (proform) and prodomain are indicated. (d) Saos cells either non-infected or infected as in $\mathrm{A}$ for $24 \mathrm{~h}$ were extracted in caspase assay buffer and assayed for caspase activity with Ac-IETD-AFC

E2 and caspase 8 during the first $24 \mathrm{~h}$ post-transfection, before the occurrence of cell death. Coexpression of caspase 8 with GFP-E2, or with an untagged version of HPV18 E2, induced a massive redistribution of the two proteins into cytoplasmic filaments (Figure $4 a$ and b and Supplementary Figure S1B). Filament formation was accompanied by translocation of both GFP-E2 and caspase 8 proteins from the detergent soluble fractions to the insoluble fractions where caspase cleavage was detected (Figure 4d). Formation of insoluble filaments only occurred when the propapoptotic HR-HPV-18 and -16 E2 proteins were coexpressed with caspase 8 , and not with the non-apoptotic HPV-6 and -11 E2 proteins, pointing out to a clear correlation between the E2-induced apoptosis and filament formation (Figure $4 \mathrm{c}$ and d). High-risk E2 proteins and caspase 8 were present in similar quantities in the insoluble fractions, indicating that E2 could play a structural role in the formation of filaments (Figure 4d). 

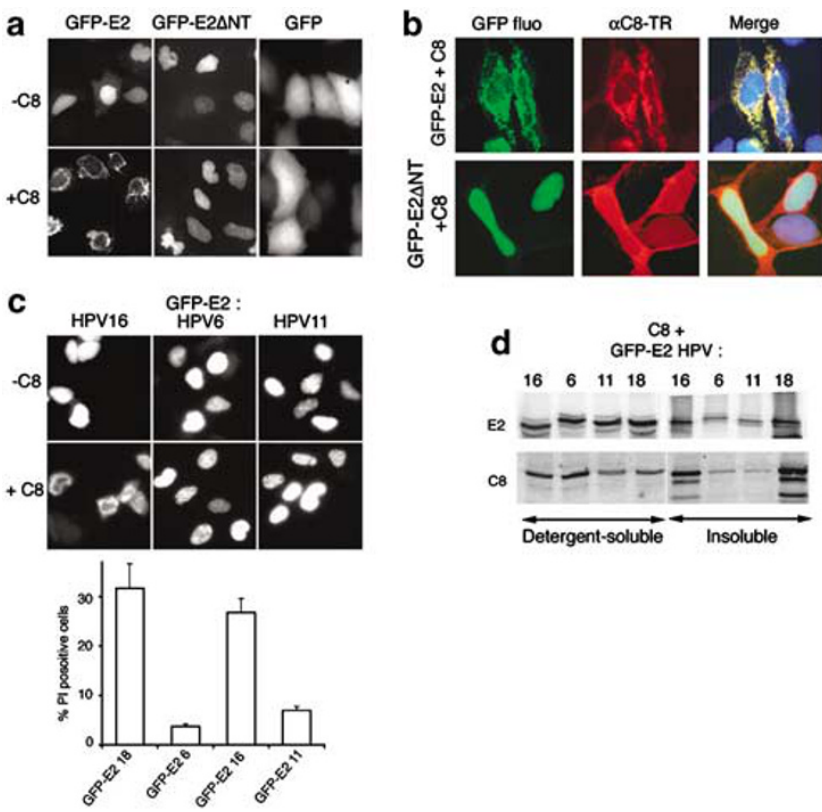

Figure 4 Assembly of the filaments containing E2 and caspase 8 correlates with E2 proapoptotic activity. (a) GFP fluorescence of 293 living cells $24 \mathrm{~h}$ after transfection with expression plasmids for GFP fusion proteins as indicated, in the presence or absence of a plasmid expressing caspase 8. (b) 293 cells cotransfected as indicated were processed $24 \mathrm{~h}$ post-transfection for caspase 8 immunofluorescence. (c) GFP fluorescence of 293 living cells $24 \mathrm{~h}$ after transfection with expression plasmids for GFP-E2 fusion proteins as indicated with or without caspase 8 expression plasmid. Viability of GFP-positive cells expressing the indicated GFP-E2 proteins were quantified by propidium iodide uptake. (d) Cells coexpressing caspase 8 and the different GFP-E2 as indicated were lyzed in 1\% Triton P300 buffer, Western blots were performed with anti-GFP or anti-caspase 8 antibodies with equivalent fractions of detergent-soluble and insoluble extracts
HPV18 E2 protein induces caspase 8 oligomerization within filaments independently of FADD through direct interaction. Analysis of the crystal structure of the aminoterminal domain of the HPV16 E2 protein did not reveal the presence of an obvious DFD typically composed of six $\alpha$-helices structures, ${ }^{14}$ indicating that E2 may not act as a typical DFD adaptor to induce caspase 8 activation. We therefore investigated whether caspase oligomerization within filaments in the presence of E2 could be mediated by the endogenous DFD adaptor FADD, which should then be found in the insoluble filaments together with E2 and caspase 8. In contrast, endogenous FADD was found exclusively in the soluble fractions of 293 cells (Figure 5a) as well as of Saos cells (not shown). These results indicated that the E2-mediated caspase 8 oligomerization into insoluble filaments is independent of the endogenous FADD. Accordingly, the silencing of the endogenous FADD using specific siRNA did not affect the level of E2-induced apoptosis, in contrast to the silencing of endogenous caspase 8 (Figure 2). As FADD was not required for caspase 8 oligomerization, we investigated whether HPV18 E2 could directly bind to caspase 8 in GST pull-down experiments. These experiments showed that HPV18 E2 binds to the caspase 8 prodomain containing tandem DEDs $A$ and $B$, and not with the caspase catalytic domain (CD) alone, whereas it only weakly binds to the DED-containing proteins VFLIP MC159 or FADD (Figure 5b). This was confirmed in co-immunoprecipitation experiments performed with co-transfected cells, where HPV18 E2 interacts efficiently with caspase 8 and not with MC159 (Figure 5c). These results show that HPV18 E2 is involved in direct, nonhomotypic interactions with the DEDs of caspase 8 , and that,

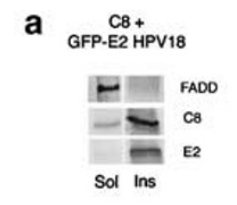

C

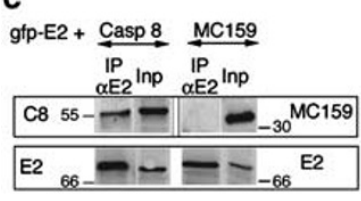

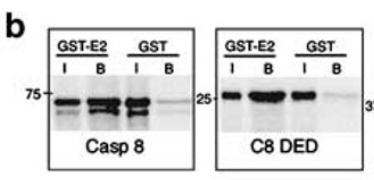
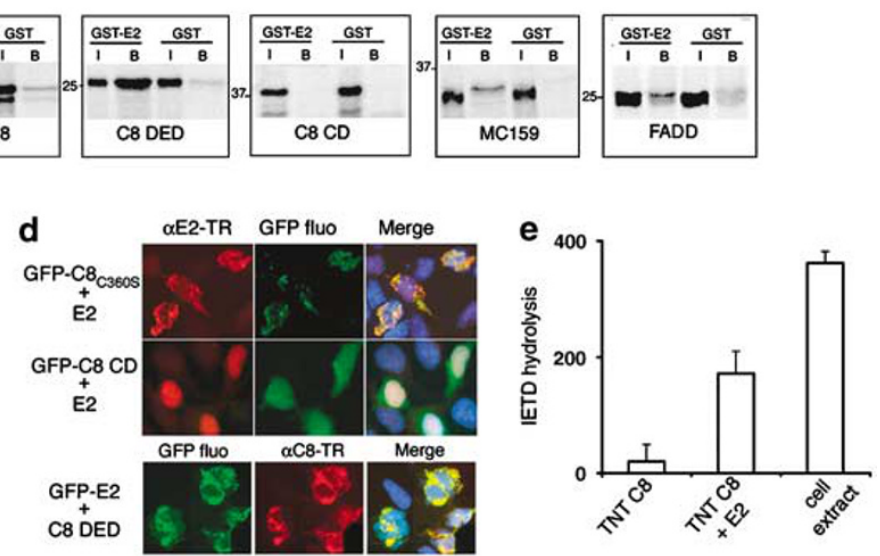

Figure 5 Filament formation is mediated through direct binding of E2 to the DED-containing prodomain of caspase 8. (a) 293 cells coexpressing caspase 8 and GFP-E2 HPV18 were lyzed in 1\% Triton P300 buffer, Western blots were performed with anti-GFP, anti-caspase 8 and anti-FADD antibodies with equivalent fractions of detergentsoluble (Sol) and insoluble (Ins) extracts. (b) GST pull-down of in vitro translated ${ }^{35}$ S-labelled proteins as indicated with GST or GST-E2 fusion proteins. Bound (B) and 1/10th input (I) ${ }^{35}$ S-labelled proteins were loaded on SDS-PAGE and revealed by autoradiography. (c) Extracts from 293 cells co-transfected with GFP-E2 and caspase 8 or GFP-E2 and HA-MC159 were immunoprecipitated with anti-E2 antibodies. Anti-E2 immunoprecipitates (IP $\alpha$ E2) and 1/10 whole cell extracts (Inp) were processed for immunoblotting with caspase 8 or HA (for MC159), and GFP antibodies for E2. (d) 293 cells transfected as indicated were processed for anti-E2 (upper panels) or anti-caspase 8 (lower panel) immunofluorescence. Spontaneous cell death was avoided in C8-DED and GFP-C8 CD expressing cells by adding Z-VAD-fmk (40 $\mu$ M), whereas the mutation in GFP-C 8 C360S prevented death induction. (e) In vitro caspase 8 activation by E2. Caspase 8 enzymatic activity was assayed on anti-E2 co-immunoprecipitation of in vitro co-translated caspase 8 and E2 (TNT C8 + E2). To ensure that the enzymatic activity was not due to some degree of caspase oligomerization induced by the immunoprecipitation, caspase assay was performed in parallel on an in vitro translated caspase 8 immunoprecipitated with anti-caspase 8 antibody (TNT C8). Caspase 8 enzymatic activity was measured, compared to $2.5 \mu \mathrm{l}$ (approximately $5 \mu \mathrm{g}$ proteins) of GFP-E2 expressing cells extract for comparison 
as expected, they are involved in filament formation (Figure 5d and Supplementary Figure S3). In addition, IETDase activity could be detected with caspase 8 coimmunoprecipitated with E2 from in vitro TNT co-translation of both proteins, suggesting that binding of E2 to caspase 8 might be sufficient to induce caspase activation (Figure $5 e$ ).

E2/caspase 8 interactions are antagonized by the MC159 vFLIP protein but not by FADD. We showed that the assembly of GFP-E2/caspase 8 filaments occurs through direct binding of E2 to the DED-containing prodomain of caspase 8 , which is the domain involved in caspase recruitment by FADD. However, GFP-E2/caspase 8 interactions are not homotypic as E2 lacks a DFD. We thus decided to investigate whether DED-mediated interactions could interfere with the binding of E2 to caspase 8. For that purpose, we used the viral antiapoptotic FLIP MC159 and the FADD adaptor, both of which are involved in DED-homotypic interactions with caspase $8 .^{15,16}$ However, while FADD is an activator, MC159 negatively interferes with caspase 8 activation through competitive binding to the DEDs of caspase 8 and of FADD. We found that filament formation did not occur in the presence of MC159, either with the endogenous caspase 8 (Figure $1 \mathrm{~b}$ ) or with transfected caspase 8 (Figure 6a), and that caspase 8 has reverted to a diffuse distribution, while the E2 protein has shuttled back to the nucleus (Figure $6 \mathrm{a}$ and $1 \mathrm{~b}$ ). Addition of in vitro translated MC159 reduced E2 binding to caspase 8 in GST pull-down experiments (Figure 6c), whereas it
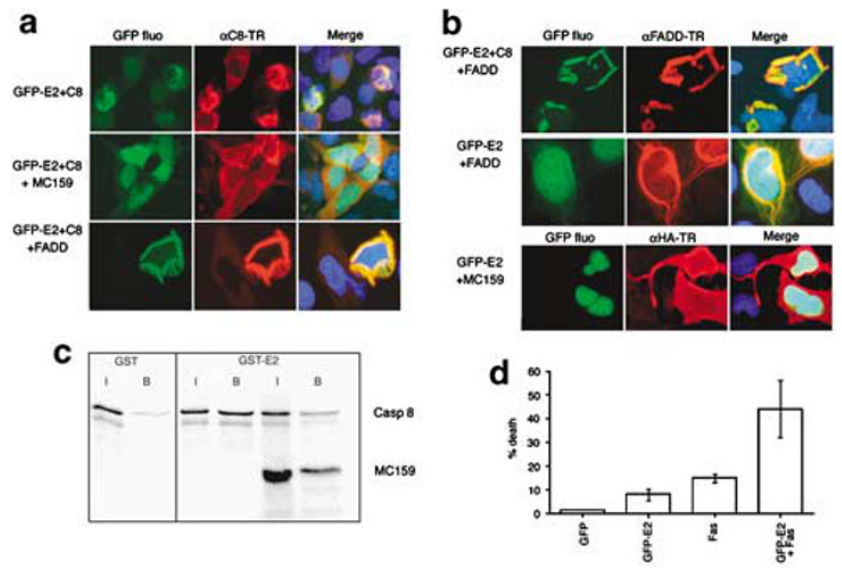

Figure 6 Filament formation is inhibited by MC159 but is compatible with FADD. (a) 293 cells were co-transfected with GFP-E2, caspase 8, HA-MC159 and FADDexpression plasmids as indicated and were processed for caspase 8 immunofluorescence (TR) $24 \mathrm{~h}$ post-transfection. (b) A total of 293 cells were co-transfected as indicated and were processed for immunofluorescence $24 \mathrm{~h}$ post-transfection with anti-FADD antibodies or anti-HA antibodies to detect HA-MC159. (c) GST pulldown experiments were performed as in Figure 5 , with a preincubation of ${ }^{35} \mathrm{~S}$ TNTgenerated caspase 8 and MC159 for $1 \mathrm{~h}$ before addition of GST-E2. (d) E2 enhances Fas-induced cell death. 293 cells were transfected with expression plasmids as indicated. Note that the amount of expression plasmid for E2 was twice less than in the other experiments in 293 cells $(1 \mu \mathrm{g})$ to reduce the level of apoptosis due to $\mathrm{E} 2$ alone, so that the effects on Fas-induced cell death can be detected. For Fas-induced cell death, the agonistic APO 1-3 anti-Fas antibody $(2 \mu \mathrm{g} / \mathrm{ml})$ was added for $16 \mathrm{~h}$. Dead cells were quantified by propidium iodide uptake $48 \mathrm{~h}$ after transfection co-immunoprecipitates efficiently with caspase 8 (Supplementary figure 4), indicating a direct competitive binding of MC159 and E2 to caspase 8. We concluded that MC159 interferes with E2 binding to caspase 8, thereby inhibiting formation of the E2/caspase8 filaments, and apoptosis.

When ectopically expressed, FADD spontaneously selfoligomerizes to form filaments, named 'death effector filament' (DEF), as previously described. ${ }^{13}$ We could show that when FADD is co-transfected with GFP-E2 and caspase 8, filaments containing the three proteins can be formed (Figure $6 \mathrm{a}$ and $\mathrm{b}$ ). When only E2 and FADD are cotransfected, however, FADD filaments marginally recruit GFP-E2, which remained mainly nuclear (Figure 6b), as expected from the weak in vitro binding of FADD to E2 (Figure $5 \mathrm{~b}$ ). We therefore concluded that, in contrast to MC159, the DED homotypic interactions of FADD with caspase 8 do not compete with interactions of E2 with caspase 8 . As the endogenous FADD is not required for the formation of E2/caspase 8 filaments or for E2-induced cell death (Figures 2 and 5), this suggests that E2 and FADD may concurrently induce the oligomerization of caspase 8 through distinct and independent mechanisms. Accordingly, HPV18 E2 is able to enhance the apoptosis induced by Fas, which involves FADD-mediated caspase 8 oligomerization, probably by cooperating with FADD to induce caspase 8 activation (Figure 6d).

A short $\alpha$-helix of the E2 amino-terminal domain mediates filament formation and cell death. The formation of filaments containing $E 2$ and caspase 8 requires the caspase 8 DEDs and the amino-terminal a
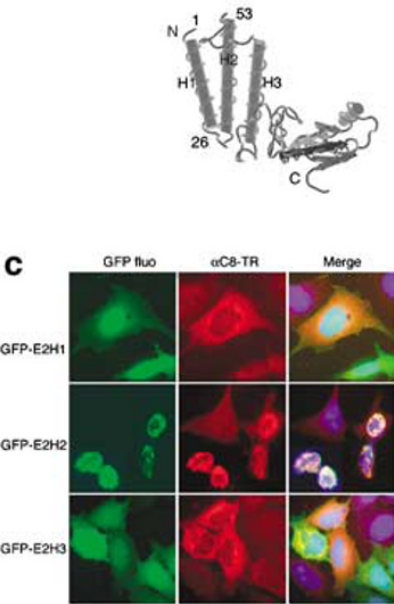

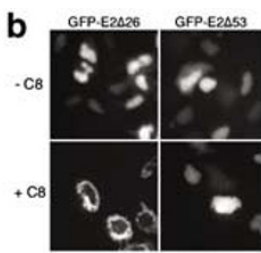

d

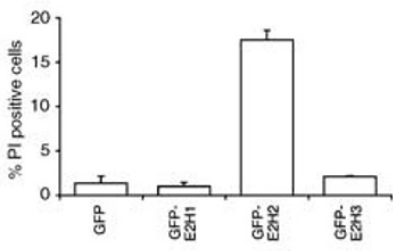

Figure 7 The second $\alpha$-helix of the amino-terminal domain of E2 recapitulates E2-induced filament formation and cell death. (a) Schematic representation of the crystal structure of the HPV16 E2 amino-terminal domain. ${ }^{14}$ (b) GFP fluorescence of 293 living cells 24 post-transfection with expression plasmids for GFP fusions with $\mathrm{N}$ terminal truncated E2 proteins starting at aa 27 (E2 $\Delta 26)$ or at aa 54 (E2 $\Delta 53)$, with or without coexpressed caspase 8 . (c) Anti-caspase 8 immunofluorescence analysis of 293 cells coexpressing caspase 8 with each of the three E2 amino-terminal $\alpha$-helices as GFP fusion proteins. (d) Viability of 293 cells expressing each GFP-E2 $\alpha$-helix as indicated, determined $48 \mathrm{~h}$ post-transfection by propidium iodide uptake 
domain of the E2 protein. To gain further insight into the type of interaction involving E2 and caspase 8, we introduced serial deletions of the $\alpha$-helices of the amino-terminal domain in the HPV18 E2 protein, according to the crystal structure (Figure 7a). A HPV18 E2 protein lacking the first $\alpha$-helix (GFP-E2 26 ) could still efficiently form cytoplasmic filaments when co-transfected with caspase 8 , whereas a protein lacking the two amino-terminal $\alpha$-helices (GFP-E2 $\Delta 53$ ) could not (Figure 7b), indicating that the second $\alpha$-helix of E2 is required for filament formation. An amino-acid sequence spanning only the second $\alpha$-helix (amino acids 27-53) fused to the GFP, assembled into filaments with caspase 8 , while the two peptides, corresponding either to the first or to the third $\alpha$-helices of E2 amino-terminal domain, did not (Figure 7c). In agreement with filament formation, the second $\alpha$-helix of E2 alone was sufficient to induce apoptosis (Figure 7d). Altogether, our results indicate that the proapoptotic activity of HR-HPVE2 proteins is based on a novel type of caspase 8-activating interaction, involving only their second $\mathrm{N}$-terminal $\alpha$-helix.

\section{Discussion}

The E2 proteins are essential multifunctional proteins involved in the control of papillomavirus transcription and replication, as well as in long-term maintenance of the viral genomes in dividing cells. A major role of HR-HPV E2 proteins, as transcription factors, is repression of the transcription of the viral E6 and E7 oncogenes by binding to their promoter sequences. This function of E2 is alleviated by disruption of the E2 gene by integration of part of the viral genome in the cellular genome during HPV-associated carcinogenesis. The E2 proteins also target the viral helicase to the viral origin of replication to activate replication of the viral genome in vivo, and they tether the viral genomes to cellular mitotic chromatin, notably through interactions with the BrD4 protein, to allow proper segregation of the viral genomes during cell division. ${ }^{17}$ HR-HPV E2 proteins can also directly influence the host cell biology, independently of their binding to the viral genome and of their transcriptional properties. ${ }^{5,18}$ One of such E2 activities is the induction of apoptosis, which was found specific of the E2 proteins of HR-viruses. ${ }^{7}$ The fact that only the HR-HPV E2 proteins have evolved a proapoptotic activity suggests that this activity could compensate for some of the transforming functions of the E6 and E7 oncogenes. In agreement with this hypothesis, mild caspase activation in non-apoptotic conditions has recently been described to play a role in the HR-HPV vegetative cycle in organotypic cell cultures, by increasing viral DNA replication. ${ }^{19}$ The E6 and E7 proteins of HR-HPV interfere with cell cycle exit and block cellular differentiation, which are required for the viral vegetative cycle. The activation of caspase 8 by E2 could therefore be required to overcome these effects and could be an alternative way for E2 to participate to the tight control of the HR-HPV E6 and E7 oncoproteins. During the course of natural infection, the role of HR-HPV E2 activation of caspase 8 may be mainly to sensitize cells to death receptor-mediated apoptosis by collaborating with FADD, as shown in this study, thereby directly counteracting the effect of the E6 oncogene that protects cells against Fas apoptosis through induction of FADD degradation. ${ }^{2}$

It is difficult to assess apoptosis in natural lesions, as apoptotic bodies are rapidly swallowed by the immune cells. However, in specific conditions of impaired immunity, as in HIV-infected women, a strong increase in the apoptotic index together with caspase 8 activation was reported in cervical intraepithelial neoplasia associated with HR-HPV, compared to similar lesions in immuno-competent women. In contrast, the apoptotic index was only slightly increased in LR-HPVassociated condyloma accuminata lesions of HIV-infected women, corroborating the link between HR-HPV-associated lesion and apoptotic induction. ${ }^{20}$ These data point to a potential involvement of apoptosis in the natural course of infection with HR-HPV. In addition, such apoptotic activity may stimulate the cellular immune response, and therefore account for the relatively high rate of spontaneous regression of HPV-associated lesions. The proapoptotic activity of HRHPV E2 proteins may also favor the clonal selection of HRHPV-infected cells lacking E2 after integration of the HPV genome and disruption of the E2 gene, this event leading to upregulated expression of the viral E6 and E7 oncogenes.

The purpose of this study was to decipher the mechanism by which HR-HPVE2 proteins induce apoptosis when located in the cytoplasm, and how E2 was able to activate caspase 8. We found that proapoptotic HR-HPV E2 proteins induce apoptosis by forming filaments containing the endogenous caspase 8 , which is then efficiently self-activated. Previously described proapoptotic filaments called 'death effector filaments' are formed when FADD is ectopically expressed, containing both FADD and activated caspase $8 .^{13}$ The filamentous structures described here are thus equivalent intracellular signaling complexes used by the viral HR-HPV E2 proteins to induce apoptosis. Activation of apical caspases comes from their oligomerization achieved through homotypic interactions with adaptors containing DFD domains, such as FADD for caspase $8 .^{12,16,21}$ Only few adaptors for caspase 8 activation have been described so far, and they all contain DED or pseudo-DEDs. ${ }^{10,22}$ We showed here that the E2 protein directly binds to the DEDs of caspase 8 , not involving a 'death fold domain,' as the motif of E2 required for this interaction could be restricted to 27 amino acids. Caspase 8 co-expression with E2 greatly stimulates filaments formation, suggesting that caspase 8 plays an active role in filament assembly rather than being recruited to pre-existing E2 filaments. There are no evidence that E2 could oligomerize through its second $\alpha$-helix ${ }^{14,23,24}$ and we therefore favour the hypothesis that E2 binding to caspase 8 would induce conformational changes enabling self-oligomerization of the associated caspase 8 , probably through its prodomain. In agreement with this hypothesis, overexpression of caspase 8 alone did not allow formation of filaments. However, it has been shown that when expressed separately, the DEDcontaining prodomain of caspase 8 can form filaments through the most internal DED $B$, whereas the $\mathrm{N}$-terminal DED A cannot. $^{13}$ We speculate that E2 binding to the prodomain of caspase 8 induces a switch from a 'closed' conformation, typical of the caspase proform, toward an 'open' conformation in which the caspase DED B would be accessible for self-oligomerization. 
We did not find colocalization of the E2/caspase 8 filaments with organelles, such as Golgi, ER, or mitochondria (Supplementary Figure S5), although we cannot exclude that other cytoplamic structures are involved. Nonetheless, detection of caspase 8 enzymatic activity of caspase 8/E2 complexes made in vitro suggests that the interaction between the two proteins is sufficient for caspase activation, even though the formation of filaments in vivo may involve additional partners. The HR-HPV E2 proteins manipulate the caspase 8 cell death pathway in a new way, opposite of the antiapoptotic viral FLIP MC 159, which inhibits caspase 8 recruitment to the DISC through DED-mediated competitive binding. The finding that non-DFD proteins, such as E2, could induce caspase 8 activation through direct interactions opens up new avenues in the search for novel types of non-death fold proapoptotic inducers.

\section{Materials and Methods}

Expression plasmids and reagents. Anti-E2 antibodies, E2 and GFP-E2 expression plasmids are as described. ${ }^{7,25}$ RFP-E2 was obtained by inserting the RFP ORF (from pDSRed2) in place of the GFP ORF in the GFP-E2 expression plasmid. E2 helices $\mathrm{H} 1$ (aa 1-26), $\mathrm{H} 2$ (aa 27-53) or H3 (aa 54-88) corresponding to the three first $\alpha$-helices of the amino terminal of HPV18 E2 as determined by the crystal structure ${ }^{14}$ were cloned by a conventional PCR-based technique in frame with GFP. The caspase 8 prodomain (aa 1-181) was cloned in pcDNA, DED A (aa 1-93) DED B (aa 94-181) were cloned in frame with GFP and caspase domain CD (aa180-480) in frame with a N-terminal FLAG tag in pcDNA 3. The cDNA encoding the wild-type caspase 8 cloned in pcDNA was provided by M Peter, expression vectors for GFP-caspase $8_{\mathrm{C} 360 \mathrm{~s}}$, GFP-C8CD and HA-MC159 by R Siegel, FADD and crmA by V Dixit and Fas by R Kitsis. Caspase 8 antibodies used in all the experiments were rabbit antibodies directed against the prodomain (Pharmingen 55993). Other antibodies were as follows: anti-FADD (Pharmingen 556402), APO 1-3 anti-Fas (Alexis Biochemicals), anti-GFP (Torrey Pines Biolabs TP401), anti-HA (Sigma H9658).

Cell transfection and infection. Cells were transfected by the calcium phosphate co-precipitation method in six-well plates with 1-4 $\mu \mathrm{g}$ of E2-expressing plasmids, $0.5 \mu \mathrm{g}$ of caspase $8, \mathrm{FADD}$ or Fas-expressing plasmids, $1 \mu \mathrm{g}$ of $\mathrm{HA}$ MC159 or crmA-expressing plasmids. Saos cells were infected at a multiplicity of infection of 500 with recombinant adenoviruses Ad GFP-E2 or Ad GFP-E2 $\Delta N T$. In these conditions GFP fusion proteins are expressed in all the cells, and GFP-HPV18 E2 induces cell death in $50 \%$ of the cells $24 \mathrm{~h}$ post-infection.

Immunofluorescence. Cells grown on coverslips were rinsed in PBS, fixed in $2 \%$ paraformaldehyde, permeabilized with $0.1 \%$ Triton, saturated in PBS- $10 \%$ SVF and incubated for $1 \mathrm{~h}$ with primary antibodies as indicated, then with the appropriate conjugated antibodies. Nuclei were stained with DAPI, $0.15 \mu \mathrm{g} / \mathrm{ml}$. Pictures were taken with a CCD camera coupled to a Zeiss Axiophot microscope, $24-48 \mathrm{~h}$ posttransfection or infection.

Video microscopy. Video microscopy was performed on recombinant adenovirus-infected Saos cells from 7 to $25 \mathrm{~h}$ post-infection with an Axiovert Zeiss microscope. Images selected at the given time points were processed by the Metamorph software.

GST pull-down experiments. Equivalent amounts of purified GST-E2 or GST were incubated for $1 \mathrm{~h}$ with ${ }^{35} \mathrm{~S}$ in vitro translated proteins produced in TNT, in binding buffer (10 mM HEPES (pH 7.7), $200 \mathrm{mM} \mathrm{NaCl}, 1 \mathrm{mM} \mathrm{MgCl}, 2 \mathrm{mM}$ DTT, $50 \mathrm{mM}$ sucrose, $0.1 \%$ Triton $X-100$ ). Beads were washed three times with binding buffer containing $1 \%$ Triton; bound proteins were separated by SDS-PAGE and revealed by autoradiography. In Figure $3 c,{ }^{35} S$ TNT-produced caspase 8 and MC159 were mixed in binding buffer, $1 \mathrm{~h}$ before addition of GST-E2, and the binding of caspase 8 alone was performed after $1 \mathrm{~h}$ incubation with TNT lysate alone.

Western-blots and immunoprecipitation. Cells were extracted in $1 \%$ Triton P300 buffer (20 mM NaH $2 \mathrm{PO}_{4}, 300 \mathrm{mM} \mathrm{NaCl}, 5 \mathrm{mM}$ EDTA, $5 \mathrm{mM}$ DTT) supplemented with protease inhibitors (Roche Diagnostics) for $30 \mathrm{~min}$ at $4{ }^{\circ} \mathrm{C}$ followed by centrifugation for $30 \mathrm{~min}$ at 13000 r.p.m. Pellets containing Tritoninsoluble material were denatured in $5 \mathrm{X}$ Laemmli sample buffer and boiled for $20 \mathrm{~min}$. Equivalent fractions of soluble and insoluble material in $1 \mathrm{X}$ sample buffer were separated on a SDS-PAGE and processed for immunoblotting. For immunoprecipitations, equal amounts of soluble proteins were immunoprecipitated with anti-E2 polyclonal rabbit antibodies, the immunoprecipitates were washed 3 times in P300 buffer containing $0.1 \%$ Triton, and processed for Western blotting. ${ }^{35}$ S-TNT produced proteins were immunoprecipitated after addition of 4 volumes of GST pull-down buffer or caspase assay buffer, with rabbit anti-C8 or anti E2 antibodies, followed by three washes in the binding buffer used for IP.

Cell viability analyses by flow cytometry. Cells were trypsinized, washed, resuspended in PBS-10\% serum containing $25 \mu \mathrm{g} / \mathrm{ml}$ propidium iodide (PI; Sigma) and analyzed by flow cytometry for GFP fluorescence and PI content. Percent of dead cells are the \% of GFP and PI-positive cells /GFP-positive cells.

siRNA-mediated gene silencing. Caspase 8 siRNA (target $5^{\prime}$-GAGCCTG CTGAAGATAATC- $3^{\prime}$ ) and FADD siRNA (target $5^{\prime}$-GAAGACCTGTGTGCAGCA $T-3^{\prime}$ ) were synthesized by Sigma. GAPDH and luciferase siRNA (Dharmacon) were used as controls. $100 \mathrm{pM}$ of siRNA were co-transfected with $4 \mu \mathrm{g}$ of GFP-E2expression plasmid in HeLa cells.

Caspase 8 activity assay. Cells were extracted in caspase assay buffer (20 mM Hepes pH 7.7, $100 \mathrm{mM} \mathrm{NaCl}, 10 \mathrm{mM}$ DTT, $1 \mathrm{mM}$ EDTA, 0.1\% CHAPS, $10 \%$ sucrose) containing $0.5 \%$ NP40. Caspase activity was measured in crude extracts containing 20-30 $\mu$ p proteins with $0.002 \mu \mathrm{M}$ of Ac-IETD-AFC in $100 \mu$ l of caspase assay buffer for $1 \mathrm{~h}$ at $37^{\circ} \mathrm{C}$. AC-IETD hydrolysis was measured by monitoring emission at $510 \mathrm{~nm}$ on excitation at $405 \mathrm{~nm}$. For in vitro caspase assay, protein $A$ beads bound to immunoprecipitated E2 and caspase 8 or caspase 8 alone produced in TNT were mixed in $100 \mu \mathrm{l}$ of assay buffer containing $0.002 \mu$ mole Ac-IETD and incubated for $1 \mathrm{~h}$ at $37^{\circ} \mathrm{C}$.

Acknowledgements. We thank Moshe Yaniv and Jonathan Weitzman for critical reading of the paper. We thank Richard Siegel, Marcus Peter, Visha Dixit and Richard Kitsis for providing expression plasmids.

This work was supported by the Pasteur Institute, CNRS, Association pour la Recherche sur le Cancer (ARC) and La Ligue Contre le Cancer.

1. Scheffner M, Huibregtse JM, Vierstra RD, Howley PM. The HPV16 E6 and E6-AP complex functions as ubiquitin-protein ligase in the ubiquitination of p53. Cell 1993; 75: 495-505.

2. Filippova M, Parkhurst L, Duerksen-Hughes PJ. The human papillomavirus 16 E6 protein binds to Fas-associated death domain and protects cells from Fas-triggered apoptosis. J Biol Chem 2004; 279: 25729-25744.

3. Jones DL, Thompson DA, Munger K. Destabilization of the RB tumor suppressor protein and stabilization of p53 contribute to HPV type 16 E7-induced apoptosis. Virology 1997; 239: $97-107$

4. Blachon S, Demeret $\mathrm{C}$. The regulatory E2 proteins of human genital papillomaviruses are pro-apoptotic. Biochimie 2003; 85: 813-819.

5. Demeret C, Garcia-Carranca A, Thierry F. Transcription-independent triggering of the extrinsic pathway of apoptosis by human papillomavirus (HPV) 18 E2 protein. Oncogene 2003; 22: 168-175.

6. Desaintes C, Demeret C, Goyat S, Yaniv M, Thierry F. Expression of the papillomavirus E2 protein in HeLa cells leads to apoptosis. EMBO J 1997; 16: 504-514.

7. Blachon S, Bellanger S, Demeret C, Thierry F. Nucleo-cytoplasmic shuttling of high-risk HPV E2 proteins induces apoptosis. J Biol Chem 2005; 280: 36088-36098.

8. Weber $\mathrm{CH}$, Vincenz $\mathrm{C}$. The death domain superfamily: a tale of two interfaces? Trends Biochem Sci 2001; 26: 475-481.

9. Shiozaki EN, Chai J, Shi Y. Oligomerization and activation of caspase-9, induced by Apaf-1 CARD. Proc Natl Acad Sci USA 2002; 99: 4197-4202. e-pub ahead of print 19 March 2002.

10. Kumar S, Colussi PA. Prodomains-adaptors-oligomerization: the pursuit of caspase activation in apoptosis. Trends Biochem Sci 1999; 24: 1-4.

11. Muppidi JR, Lobito AA, Ramaswamy M, Yang JK, Wang L, Wu H et al. Homotypic FADD interactions through a conserved RXDLL motif are required for death receptor-induced apoptosis. Cell Death Differ 2006; 13: 1641-1650.

12. Carrington PE, Sandu C, Wei Y, Hill JM, Morisawa G, Huang T et al. The structure of FADD and its mode of interaction with procaspase-8. Mol Cell 2006; 22: 599-610. 
13. Siegel RM, Martin DA, Zheng L, Ng SY, Bertin J, Cohen J et al. Death-effector filaments: novel cytoplasmic structures that recruit caspases and trigger apoptosis. J Cell Biol 1998 141: $1243-1253$.

14. Antson AA, Burns JE, Moroz OV, Scott DJ, Sanders CM, Bronstein IB et al. Structure of the intact transactivation domain of the human papillomavirus E2 protein. Nature 2000; 403 805-809.

15. Garvey TL, Bertin J, Siegel RM, Wang GH, Lenardo MJ, Cohen Jl. Binding of FADD and caspase-8 to molluscum contagiosum virus MC159 v-FLIP is not sufficient for its antiapoptotic function. J Virol 2002; 76: 697-706.

16. Medema JP, Scaffidi C, Kischkel FC, Shevchenko A, Mann M, Krammer PH et al. FLICE is activated by association with the CD95 death-inducing signaling complex (DISC). EMBO J 1997: 16: 2794-2804.

17. McBride AA, Oliveira JG, McPhillips MG. Partitioning viral genomes in mitosis: same idea different targets. Cell Cycle 2006; 5: 1499-1502.

18. Bellanger S, Blachon S, Mechali F, Bonne-Andrea C, Thierry F. High-risk but not low-risk HPV E2 proteins bind to the APC activators Cdh1 and $\mathrm{Cdc} 20$ and cause genomic instability. Cell Cycle 2005; 4: 1608-1615.

19. Moody CA, Fradet-Turcotte A, Archambault J, Laimins LA. Human papillomaviruses activate caspases upon epithelial differentiation to induce viral genome amplification. Proc Natl Acad Sci USA 2007; 104: 19541-19546.
20. Walker $\mathrm{F}$, Adle-Biassette $\mathrm{H}$, Madelenat $\mathrm{P}$, Henin $\mathrm{D}$, Lehy $\mathrm{T}$. Increased apoptosis in cervical intraepithelial neoplasia associated with HIV infection: implication of oncogenic human papillomavirus, caspases, and Langerhans cells. Clin Cancer Res 2005; 11: 2451-2458.

21. Chang DW, Ditsworth D, Liu H, Srinivasula SM, Alnemri ES, Yang X et al. Oligomerization is a general mechanism for the activation of apoptosis initiator and inflammatory procaspases. J Biol Chem 2003; 278: 16466-16469.

22. Gervais FG, Singaraja R, Xanthoudakis S, Gutekunst CA, Leavitt BR, Metzler M et al. Recruitment and activation of caspase- 8 by the Huntingtin-interacting protein Hip-1 and a novel partner Hippi. Nat Cell Biol 2002; 4: 95-105.

23. Abbate EA, Voitenleitner $C$, Botchan MR. Structure of the papillomavirus DNA-tethering complex E2:Brd4 and a peptide that ablates HPV chromosomal association. Mol Cell 2006; 24: 877-889.

24. Wang Y, Coulombe R, Cameron DR, Thauvette L, Massariol MJ, Amon LM et al. Crystal structure of the E2 transactivation domain of human papillomavirus type 11 bound to a protein interaction inhibitor. J Biol Chem 2004; 279: 6976-6985.

25. Bellanger S, Demeret C, Goyat S, Thierry F. Stability of the human papillomavirus type 18 E2 protein is regulated by a proteasome degradation pathway through its amino-terminal transactivation domain. J Virol 2001; 75: 7244-7251.

Supplementary Information accompanies the paper on Cell Death and Differentiation website (http://www.nature.com/cdd) 